- Clinical Research •

\title{
Short-term efficacy of cetuximab combined with radiotherapy or chemotherapy on head and neck cancer: a report of 11 cases
}

\author{
Liang-Ping Xia, ${ }^{1,2}$ Bei Zhang, ${ }^{1,2}$ Mao-Zhen Liu, ${ }^{1,2}$ Pi-Li Hu, ${ }^{1,2}$ Xu-Xian Chen , ${ }^{1,2}$ \\ Gui-Fang Guo, ${ }^{1,2}$ Hui-Juan Qiu , ${ }^{1,2}$ Yu-Ming Rong, ${ }^{1,2}$ Sui-Yi Qian, ${ }^{1,2}$ \\ Fei-Fei Zhou,,$^{1,2}$ Yuan-Yuan Huang ${ }^{1,2}$ and Tao-Li Wang ${ }^{1,2}$
}

\author{
1. State Key Laboratory of \\ Oncology in South China, \\ Guangzhou, Guangdong, 510060, \\ P. R. China \\ 2. VIP Region, \\ Sun Yat-sen Universty Cancer \\ Center, Guangzhou, Guangdong, \\ 510060 , \\ P. R. China
}

Correspondence to : Liang-Ping Xia

Tel. : 86.20.87343107

Email : xialiangping@163.com

Grant: Major science and thechnology project of "National signicant new drug creation” (No. 2008ZX09312-002)

This paper was translated into English from its original publication in Chinese. Translated by: Guangzhou Liheng and Wei Liu on 2009-08-18

The original Chinese version of this paper is published in:Ai Zheng (Chinese Journal of Cancer) 28(9);http://www.cjesysu.cn/cn/ article.asp?id=15798)

Submitted : 2008-12-23

Revised : 2009-06-22

\begin{abstract}
[Abstract] Background and Objective: Cetuximab combined with radiotherapy or chemotherapy has been used to treat head and neck cancer in recent years, but few reports are available in China now. This study was to summarize our experiences in treating patients with head and neck cancer cetuximab. Methods: From October 1st, 2005 to September 30th, 2008, six with patients head and neck cancer were treated using cetuximab combined with radiotherapy and five were treated using cetuximab combined with chemotherapy in Sun Yat-sen University Cancer Center. The short-term efficacy and safety were analyzed. Results: A total of 82 cycles of cetuximab treatment, with a median of seven cycles, were administered safely. There was no treatment-associated death and no cetuximab-associated discontinuation. In cetuximab combined with radiotherapy group, four patients achieved complete response (CR) and two achieved partial response $(\mathrm{PR})$; all $\mathrm{CR}$ patients had hadacne-like rash (three cases were $\geqslant$ grade III), only one PR patient had grade I rash; five patients had skin reaction in the irradiation field (four cases of skin reaction were $\geqslant$ grade III ); hematological toxicity was slight excepted one case of grade IV. In cetuximab combined chemotherapy group, two patients achieved PR, two had stable disease (SD) and one had progressed disease (PD); the of acne-like rash was low, and three patients experienced bone marrow depression above grade III. Conclusion: Cetuximab combined wthe either radiotherapy or chemotherapy are good options for suitable patients with head and neck cancer.
\end{abstract}

Key words: cetuximab, head and neck neoplasm, radiotherapy, chemotherapy, efficacy, safety

Cetuximab is widely used in the treatment of squamous cell carcinoma of head and neck (SCCHN). It can be used in combination with radiotherapy ${ }^{1,2}$ or concurrent radiochemotherapy, ${ }^{3}$ used alone or with DDP as the first-line ${ }^{46}$ or second-line treatment ${ }^{7}$, 8 for recurrent or metastatic HNSCC. the cetuximab is expensive and is used in China lately, reports on the treatment of HNSCC 
with cetuximab are rare in China. Xu et al. ${ }^{9}$ reported 20 cases that had been treated with cetuximab, only three were not nasopharyngeal carcinoma (NPC). In the present study, we summarized 11 cases of SCCHN other than NPC that were treated with cetuximab combined radiotherapy and/or chemotherapy, analyzed the efficacy and adverse events to enrich experiences of treatment of SCCHN with cetuximab.

\section{Materials and Methods}

Clinical data. A total of 15 HNSCC patients were treated with cetuximab in Cancer Center of Sun Yat-sen University from October $1^{\text {st }} 2005$ to September $30^{\text {th }} 2008$. Of the 15 patients, two were excluded because of incomplete medical record, and two were excluded because they were still under treatment; The rest 11 all received cetuximab combined radiotherapy and/or chemotherapy.

Of the 11 patients, five were na $\square$ ve cases and six were recurrent cases; six were treated with cetuximab combined radiotherapy and five with cetuximab combined with chemotherapy (Table 1). Previous treatment strategies of recurrent patients are shown in Table 2. Except for one patient with adenocarcinoma, the other ten were all with squamous cell carcinomas; one had papillary thyroid carcinoma accompanied by squamous cell carcinoma. The ECOG scores of the patients before cetuximab administration were $<2$; routine blood examination, liver and renal functions were normal.

Treatment strategies. Intravenous injection of cetuximab was firstly given at a dose of 400 $\mathrm{mg} / \mathrm{m}^{2}$ for $120 \mathrm{~min}$, maintained at $250 \mathrm{mg} / \mathrm{m}^{2}$ for $60 \mathrm{~min}$ and administrated weekly. Pretreatment were performed before each cetuximab administration: intramuscular injection of 40-50 $\mathrm{mg}$ of diphenhydramine with intravenous injection of $0.4 \mathrm{~g}$ of cimetidine dissolved in $20 \mathrm{~mL}$ of normal saline. Electrocardiogram monitoring was used during cetuximab administration. Radiotherapy and chemotherapy were both adopted standard regimens.

Evaluation criteria. Short-term efficacy was evaluated by RESIST solid tumor evaluation criteria: complete remission (CR), partial remission (PR), stable disease (SD) and progressive disease (PD). CT or MRI scan were performed on all the 11 patients. Adverse events were observed and recorded according to NCI-CTC criteria.

Follow-up. The 11 patients were followed up till December $20^{\text {th }} 2008$. Five patients died.

Table 1 General data of the 11 patients with head and neck cancer

\begin{tabular}{|c|c|c|c|c|c|c|}
\hline Patient's No. & Gender & Age & Tumor site & TNM stage & Pathologic type & Notes \\
\hline 1 & Male & 84 & Hypopharyngeal carcinoma & T2N1M0 & SCC (grade II ) & Initial treatment \\
\hline 2 & Female & 73 & Hypopharyngeal carcinoma & T4N1M0 & SCC (grade II - III ) & Initial treatment \\
\hline 3 & Male & 59 & Hypopharyngeal carcinoma & $\mathrm{T} 2 \mathrm{~N} 2 \mathrm{M} 0$ & SCC (grade II ) & Initial treatment \\
\hline 4 & Female & 62 & Thyroid cancer & T4N0M0 & Papillary carcinoma and SCC (grade III ) & Initial treatment \\
\hline 5 & Male & 57 & Hypopharyngeal carcinoma & T4N2M0 & $\begin{array}{l}\text { Atypical amplification and cancergesis of } \\
\text { sequamous epithelial }\end{array}$ & Initial treatment \\
\hline 6 & Male & 45 & Hypopharyngeal carcinoma & T4N2M0 & SCC (grade II ) & Recurrent \\
\hline 7 & Male & 53 & Hypopharyngeal carcinoma & T4N2M0 & SCC (grade II ) & Recurrent \\
\hline 8 & Male & 53 & $\begin{array}{l}\text { Adenocarcinoma of external } \\
\text { auditory canal }\end{array}$ & Unclassified $^{\mathrm{a}}$ & Adenocarcinoma (grade II ) & Recurrent \\
\hline 9 & Male & 37 & Hypopharyngeal carcinoma & T4N2M0 & SCC (grade II ) & Recurrent \\
\hline 10 & Male & 66 & Hypopharyngeal carcinoma & T4N2M1 & SCC (grade I - II ) & Recurrent \\
\hline 11 & Male & 59 & Laryngeal cancer & Unclassified $^{\mathrm{b}}$ & SCC (grade II ) & Recurrent \\
\hline
\end{tabular}

${ }^{a}$ No classification is available due to tumor recurrence;

bthe initial data is unavailable since the patient accepted treatment oversea. SCC: sequamous cell carcinoma. 


\section{Results}

A total of 82 cycles of cetuximab treatment were completed with a median of 7 cycles. The patients in cetuximab combined radiotherapy group received 49 cycles of cetuximab treatment with a median of 7.5 cycles; those in cetuximab combined chemotherapy group received 33 cycles with a median of 7 cycles. The efficacy and adverse events of the 11 patients are shown in Table 3, with no treatment-related death reported. Because the study was retrospective, only cetuximab-related skin rashes, radiotherapy-related skin reactions, and chemotherapy-related hematologic toxicities were concluded.

Of the six patients treated with cetuximab combined radiotherapy, four achieved CR, and two achieved PR. Of the PR patients, one received chemotherapy induction before radiotherapy, one had papillary thyroid carcinoma accompanied by squamous cell carcinoma. The ninth CR patient had laryngopharyngeal carcinoma and refused surgery and received cetuximab plus radiotherapy, and no recurrence or metastasis was reported after 24 months treatment. The other five patients were

Table 2 Previous treatment of the six recurrent patients

\begin{tabular}{|c|c|c|c|}
\hline Patient's No. & Surgery & Radiotherapy / chemotherapy & Results \\
\hline 6 & $\begin{array}{l}\text { TL, resection of the right } \\
\text { pyriform sinus, right } \mathrm{RND}\end{array}$ & $\begin{array}{l}\text { Postoperative concomitant radiochemotherapy } \\
\text { (DDP plus 5-FU) }\end{array}$ & Failure in cervical lymph nodes \\
\hline 7 & TL, right RND & $\begin{array}{l}\text { Postoperative radiotherapy and several cycles } \\
\text { of palliative chemotherapy }\end{array}$ & $\mathrm{PD}$, lung metastasis \\
\hline 8 & tumor resection, MND & Postoperative radiotherapy & Recurrence, and received re-operations \\
\hline 9 & Left RND & $\begin{array}{l}\text { Postoperative concomitant radiochemotherapy } \\
\text { (taxol plus DDP) }\end{array}$ & Recurrence at primary site 34 months later \\
\hline 10 & No & Seven cycles of taxol plus platinum & PD \\
\hline 11 & TL & $\begin{array}{l}\text { Postoperative radiotherapy and palliative } \\
\text { chemotherapy (docetaxel, DDP plus Xeloda) }\end{array}$ & $\begin{array}{l}\text { More time recurrence in primary site and } \\
\text { lymph nodes, and received re-operations }\end{array}$ \\
\hline
\end{tabular}

TL, total laryngectomy; RND, radical neck dissection; MND, modified neck dissection; DDP, cisplatin; 5-FU, 5-fluorouracil; PD, progressed disease.

Table 3 Responses and adverse events of the 11 head and neck patients after cetuximab treatment

\begin{tabular}{|c|c|c|c|c|c|c|c|c|c|}
\hline $\begin{array}{c}\text { Patient's } \\
\text { No. }\end{array}$ & $\begin{array}{l}\text { Combined } \\
\text { treatment }\end{array}$ & $\begin{array}{l}\text { Cycles of } \\
\text { cetuximab }\end{array}$ & Response & $\begin{array}{l}\text { Response } \\
\text { duration } \\
\text { (months) }\end{array}$ & Rash & $\begin{array}{c}\text { Skin } \\
\text { reaction }\end{array}$ & $\begin{array}{c}\text { Hematological } \\
\text { toxicity }\end{array}$ & $\begin{array}{l}\text { Current } \\
\text { status }\end{array}$ & $\begin{array}{c}\text { Survival } \\
\text { time }\end{array}$ \\
\hline 1 & RT & 9 & CR & 24 & Grade IV & Grade IV & Grade II & Tumor-free & $24 / 24$ \\
\hline 2 & RCT & 4 & $\mathrm{CR}$ & 16 & Grade III & Grade III & Grade IV & Tumor-free & $16 / 16$ \\
\hline 3 & RCT & 15 & CR & 38 & Grade I & Grade IV & Grade I & Tumor-free & $38 / 38$ \\
\hline 4 & RCT & 7 & PR & 2 & Grade I & Grade II & Grade I & Died & $6 / 7$ \\
\hline 5 & CT before RT & 6 & PR & 4 & Grade 0 & Grade 0 & Grade I & Tumor-residual & $15 / 17$ \\
\hline 6 & CT & 9 & $\mathrm{SD}$ & 2 & Grade I & Grade 0 & Grade I & Died & $6 / 14$ \\
\hline 7 & CT & 4 & SD & 1 & Grade II & Grade 0 & Grade 0 & Died & $8 / 21$ \\
\hline 8 & CT & 10 & PR & 5 & Grade I & Grade 0 & Grade IV & Died & $14 / 59$ \\
\hline 9 & RT & 8 & CR & 24 & GradeIV & Grade III & Grade 0 & Tumor-free & $24 / 77$ \\
\hline 10 & CT & 3 & PR & 3 & Grade 0 & Grade 0 & Grade III & Tumor-residual & $3 / 12$ \\
\hline 11 & CT & 7 & PD & 0 & Grade I & Grade 0 & Grade III & Died & $11 / 43$ \\
\hline
\end{tabular}

RT, radiotherapy; RCT, radiochemotheapy; CT, chemotherapy. The data of survival time are presented as time from cetuximab use to last follow-up/time from diagnosis to last follow-up. 
in their initial treatment. The second to fourth patients received concurrent radiochemotherapy plus cetuximab: the third patient completed treatment of doxycycline; carboplatin plus cetuximab, the fourth completed treatment of cisplatin plus cetuximab; the second received treatment of cisplatin plus cetuximab, but discontinued treatment of cisplatin because of grade IV bone marrow suppression. Four CR patients all had acne-like rashes (three had rashes above grade III), while only one of the two PR patients had grade I rash. The five patients who received concurrent radiotherapy all had skin reactions in irradiation field (four were above grade IV). Hematological toxicity was mild in five patients except for one that had grade IV toxicity.

Of the five patients treated with cetuximab combined chemotherapy, two achieved PR, two had SD, and one had PD. Among them, the seventh, eighth and tenth patients had failed several chemotherapy regimens before cetuximab treatment, and the eighth patient achieved five months of PR after adding cetuximab to the former ineffective regimen, and the tumor progressed slowly when treated with cetuximab, Xeloda plus nedaplatin, cetuximab plus gemcitabine and cetuximab plus endostar in the following seven months. The seventh and tenth patients achieved SD and PR after using novel cytotoxic drugs plus cetuximab. The incidence of rash was not high in this group, however, three patients had bone marrow suppression over grade III.

\section{Discussion}

The overexpression of EGFR in SCCHN can inhibit the tumor cell apoptosis and increase the incidence of metastasis, therefore the tumor is more invasive and resistant to chemotherapy and radiotherapy, resulting in poor prognosis and low survival rate. ${ }^{1}$ Both in vivo and in vitro study have demonstrated that the inhibition of EGFR with monoclonal antibody or tyrosine kinase inhibitor can reverse tumor cell resistance to radiotherapy and chemotherapy. ${ }^{10}$ On the other hand, the EGFR is overexpressed in HNSCC (even 100\%), ${ }^{11}$ which suggested the importance of cetuximab in the treatment of SCCHN.

Cetuximab combined radiotherapy is more effective. Although surgical operation is the mainstay radical treatment of SCCHN, most patients have been in III or IV stage at initial evaluation. ${ }^{12}$ At this circumstance, the surgical operation alone is ineffective, and the adjuvant salvage surgery + concurrent radiotherapy or chemotherapy become the standard strategy. ${ }^{13}$ However, the considerable toxicity imposed by chemotherapy and radiotherapy urge investigators to seek drugs that are more effective and less toxic, and cetuximab is such a drug. ${ }^{1,2}$ The combination of radiotherapy and cetuximab is appropriate. The EGFR is highly expressed in head and neck tumors, which is associated with the post-radiotherapy proliferation. ${ }^{14,15}$ Furthermore, phase III clinical trial has proved that cetuximab combined radiotherapy was able to significantly prolong the survival time of the patients, which demonstrated the importance of cetuximab in head and neck tumor treatment. ${ }^{2}$ In the present study, six of the 11 patients received combined radiotherapy, and four of them achieved CR, and one case with post-radiotherapy recurrence achieved $\mathrm{CR}$ with cetuximab + radiotherapy. One feature of those received radiotherapy was the marked skin rashes, and the incidence of III/IV skin toxicity was high, ${ }^{16,17}$ which was likely to be associated with the additive effect of radiotherapy and cetuximab. Why the cetuximab combined radiotherapy achieved a better clinical outcome in the present study? Firstly, Kras gene of head and neck tumor is wild type with mutation rate of less than $5 \%,{ }^{18}$ and it is concluded from the intestinal tumor that the Kras mutation is an important indicator of resistance to cetuximab. ${ }^{19}$ Secondly, five of the six patients received radiotherapy were nasopharyngeal carcinoma which were primarily poorly differentiated squamous cell carcinoma, and the sensitivity of tumor cells to the radiotherapy is negatively associated with tumor differentiation. Finally, five of the six patients received radiotherapy were na $\square v e$ patients, and the resistance to radiotherapy and chemotherapy were rare. Currently, studies on cetuximab 
combined concurrent radiotherapy or chemotherapy are rare. ${ }^{1}$ In a phase II study conducted by Pfister DG, ${ }^{3} 15$ out of 16 cases achieved $\mathrm{CP}+\mathrm{PR}$. However, the trial was stopped because of significant side effects. Since cetuximab + radiotherapy is superior to radiotherapy alone, how about its equivalence to the concurrent chemotherapy and radiotherapy? Unfortunately, there is no head-to-head comparison study up to now. However, Caudell JJ20 retrospectively compared the efficacy of radiotherapy + chemotherapy based on DDP group (ChRT) to cetuximab+ radiotherapy group and found that three-year local control rate, non-systemic metastasis survival rate, disease-specific survival rate were similar between ExRT and ChRT groups. There were three cases in the present study that received cetuximab + concurrent radiotherapy and chemotherapy.

Cetuximab combined chemotherapy is an option for late stage patients. Median progression-free survival (PFS) time for the recurrent or metastatic SCCHN patient treated with PF strategy $(\mathrm{DDP}+5 \mathrm{Fu})$ is two months. ${ }^{21}$ Once the resistance to platinum-based chemotherapy appears, the patient may have a poor prognosis and there is no standard treatment strategy. ${ }^{5}$ Therefore, the establishment of a more effective approach is urgent, and the cetuximab, which is proved to be effective in intestinal cancer, also has some effects in the SCCHN treatment.

Firstly, cetuximab is effective for the patients who have failed the platinum based chemotherapy, that is, cetuximab is an effective second line strategy. Baselga J firstly adopted cetuximab+ platinum regimen and found that the response rate was $10 \%,{ }^{8}$ disease control rate was $53 \%$, the median time to progression (TTP) and overall survival (OS) were 85 and 183 days, respectively, which proved the effectiveness and safety of this strategy. Vermorken JB tried the clinical feasibility of cetuximab alone regimen. ${ }^{7}$ If disease progressed, the platinum was added to the cetuximab. The result showed that the efficacy of cetuximab alone was comparable to the cetuximab + platinum. In order to compare the efficacy of cetuximab alone with cetuximab+ platinum in patients with platinum resistant recurrent and metastatic HNSCC, Vermorken JB pooled analyzed three prospective study and one retrospective study, ${ }^{22}$ and found that there was no significant difference between the cetuximab alone group and cetuximab+ cisplatin/carboplatin group. The survival time of those received cetuximab was longer than those received support treatment alone and with chemotherapy alone. However, Vermorken added platinum after the patient failed cetuximab, ${ }^{7}$ so the study is not sufficient to conclude that cetuximab alone is equivalent to cetuximab+ platinum in patients with platinum resistance, but only suggested that the platinum based chemotherapy was ineffective after the induction of cetuximab resistance. Although cetuximab was used as a second or third line therapy in the cetuximab combined chemotherapy group in the present study, four cases achieved disease control $(\mathrm{PR}+\mathrm{SD})$, and two cases were unable to maintain the treatment until cancer progression because of financial problem. It is noted that the remission phase is short in our study, and $3 / 5$ patients had over III grade bone marrow suppression, 4/5 patients died, indicating that cetuximab is less beneficial in late stage.

Secondly, cetuximab combined chemotherapy is an effective first line therapy. In the phase II trial treating recurrent or metastatic SCCHN, ${ }^{4}$ the total effective rate of cetuximab+ cisplatin/carboplatin was up to $36 \%$, suggesting the feasibility of this approach as the first line therapy. Vermorken JB conducted a phase III clinical trial involving 442 patients for this strategy and found that cetuximab was able to increase the response rate $(20 \%$ and $36 \%),{ }^{6}$ PFS (3.3 and 5.6 months) and OS (7.4 and 10.1 months) significantly, and except for skin reactions, cetuximab did not had other side effects. Burtnes B investigated the cisplatin combined cetuximab strategy, ${ }^{5} 117$ patients were randomized to the cisplatin + placebo group and cisplatin+ cetuximab group. The PFS and median total survival time were improved $(\mathrm{p}=0$. 21), while the objective effective rate was significantly improved (10\% v.s 26\%, $\mathrm{P}=0.03)$, and the patients with skin rashes had survival 
priority (hazard ratio $=0.42, \mathrm{p}=0.01$ ). There was only one case in the present study that received first line therapy of cetuximab after tumor recurrence, and achieved PR.

Nontheless, because the benenifit of the patient is limited no matter the cetuximab is used as first or second line therapy, several aspects should be taken into consideration. The first one is how to predict clinical outcome, although the Kras gene is a reliable negative predictive index (the patient can not benifit from the treatment). ${ }^{19}$ The fact that the mutation rate of Kras is less than $5 \%$ in head and neck tumor remarkably compromise its predictive value. ${ }^{18}$ Rash is probably an practical predictive index, that is because there is definite association between cetuximab efficacy and rash in HNSCC patient. ${ }^{5}$ In the present study, $5 / 6$ patients with clinical benifits had rashes, and 3/4 patients with CR had skin rashes over grade III. Most patients had mild rashes in chemotherapy group. The second is the cost effectiveness. Although cetuximab is safe and effective, the head and neck tumor primarily affects the economically underdeveloped areas, so the pros and cons should be balanced before choosing the expensive cetuximab, especially for palliative therapy, and the considerable financial burden should be avoided. The third is the route of adminstration. The conventional dosage of cetuximab is 400 $\mathrm{mg} / \mathrm{m}^{2}$ for the first time, followed by maintenance with $250 \mathrm{mg} / \mathrm{m}^{2}$ per week. Some investigators have tried the dosage of $500 \mathrm{mg} / \mathrm{m}^{2}$ every other week and found no significant difference in terms of eficacy and side effect, which brought convenience to both clinicians and patients. ${ }^{23,24}$ There is no such trial in head and neck tumors, so the conventional administration method was used. The fourth is the quality of life. One merit of the cetuximab is its safety that it does not increase the toxicity of radiotherapy and chemotherapy. ${ }^{25,22}$ Further study should be carried out to develop appropriate strategy for the use of cetuximab combined chemotherapy that is more effective and less toxic.

\section{References}

[1] Merlano M, Occelli M. Review of cetuximab in the treatment of squamous cell carcinoma of the head and neck $[\mathrm{J}]$. Ther Clin Risk Manag, 2007,3 (5) : 871-876.

[2] Bonner JA, Harari PM, Giralt J, et al. Radiotherapy plus cetuximab for squamous-cell carcinoma of the head and neck [J]. N Engl J Med, 2006,354(6) :567-578.

[3] Pfister DG, Su YB, Kraus DH, et al. Concurrent cetuximab, cisplatin, and concomitant boost radiotherapy for locoregionally advanced, squamous cell head and neck cancer: a pilot phase II study of a new combined-modality paradigm $[\mathrm{J}]$. J Clin Oncol, 2006,24(7):1072-1078.

[4] Bourhis J, Rivera F, Mesia R, et al. Phase I / II study of cetuximab in combination with cisplatin or carboplatin and fluorouracil in patients with recurrent or metastatic squamous cell carcinoma of the head and neck $[\mathrm{J}]$. J Clin Oncol, 2006,24 ( 18 ) : 2866-2872.

[5] Burtness B, Goldwasser MA, Flood W, et al. Phase III randomized trial of cisplatin plus placebo compared with cisplatin plus cetuximab in metastatic/recurrent head and neck cancer: an Eastern Cooperative Oncology Group study $[\mathrm{J}]$. J Clin Oncol, 2005,23 (34) : 8646-8654.

[6] Vermorken JB, Mesia R, Rivera F, et al. Platinum-based chemotherapy plus cetuximab in head and neck cancer $[\mathrm{J}] . \mathrm{N}$ Engl J Med, 2008,359(11) : 1116-1127.

[7] Vermorken JB, Trigo J, Hitt R, et al. Open-label, uncontrolled, multicenter phase II study to evaluate the efficacy and toxicity of cetuximab as a single agent in patients with recurrent and/or metastatic squamous cell carcinoma of the head and neck who failed to respond to platinum-based therapy [J]. J Clin Oncol, 2007, 25 (16): 2171-2177.

[8] Baselga J, Trigo JM, Bourhis J, et al. Phase II multicenter study of the antiepidermal growth factor receptor monoclonal antibody cetuximab in combination with platinum-based chemotherapy in patients with platinum-refractory metastatic and/or recurrent squamous cell carcinoma of the head and neck [J]. J Clin Oncol, 2005,23 (24) :5568-5577.

[9] Xu TT, Hu CS, Ying HM, et al. Cetuximab plus other methods in the treatment of patients with head and neck squamous cell carcinomas (HNSCC) [J]. Chin Oncol, 2008,18(3):230-233. [in Chinese]

[10] Goldstein NI, Prewett M, Zuklys K, et al. Biological efficacy of a chimeric antibody to the epidermal growth factor receptor in a human tumor xenograft model $[\mathrm{J}]$. Clin Cancer Res, 1995, 1 (11) : 1311-1318.

[11] Rubin Grandis J, Melhem MF, Gooding WE, et al. Levels of TGF-alpha and EGFR protein in head and neck squamous cell carcinoma and patient survival [J]. J Natl Cancer Inst, 1998, 
$90(11): 824-832$.

[12] Vokes EE, Weichselbaum RR, Lippman SM, et al. Head and neck cancer [J]. N Engl J Med, 1993,328(3) : 184-194.

[13] Garden AS, Asper JA, Morrison WH, et al. Is concurrent chemoradiation the treatment of choice for all patients with stage III or IV head and neck carcinoma? [J]. Cancer, 2004, 100 (6) : :1171-1178.

[14] Peter RU, Beetz A, Ried C, et al. Increased expression of the epidermal growth factor receptor in human epidermal keratinocytes after exposure to ionizing radiation $[\mathrm{J}]$. Radiat Res, 1993, 136(1):65-70.

[15] Schmidt-Ullrich RK, Mikkelsen RB, Dent P, et al. Radiation-induced proliferation of the human A431 squamous carcinoma cells is dependent on EGFR tyrosine phosphorylation [J]. Oncogene, 1997, 15(10): 1191-1197.

[16] Giro C, Berger B, Bölke E, et al. High rate of severe radiation dermatitis during radiation therapy with concurrent cetuximab in head and neck cancer: results of a survey in EORTC institutes [J]. Radiother Oncol, 2009,90 (1):142143.

[17] Vano-Galvan S, de las Heras E, Harto A, et al. Severe cutaneous toxicity during concomitant radiotherapy and cetuximab treatment of head and neck cancer $[\mathrm{J}]$. Eur $\mathrm{J}$ Dermatol, 2008, 18(4):471-472.

[18] Matsuda H, Konishi N, Hiasa Y, et al. Alterations of p16/ CDKN2, p53 and ras genes in oral squamous cell carcinomas and premalignant lesions [J]. J Oral Pathol Med, 1996, 25 (5) : 232-238.

[19] Karapetis CS, Khambata-Ford S, Jonker DJ, et al. K-ras mutations and benefit from cetuximab in advanced colorectal cancer [J]. N Engl J Med, 2008,359(17) : 1757-1765.

Caudell JJ, Sawrie SM, Spencer SA, et al. Locoregionally

[20] advanced head and neck cancer treated with primary radiotherapy: a comparison of the addition of cetuximab or chemotherapy and the impact of protocol treatment $[\mathrm{J}]$. Int $\mathrm{J}$ Radiat Oncol Biol Phys, 2008,71(3) :676-681.

Jacobs C, Lyman G, Velez-Garcia E, et al. A phase III

[21] randomized study comparing cisplatin and fluorouracil as single agents and in combination for advanced squamous cell carcinoma of the head and neck [J]. J Clin Oncol, 1992,10 (2) : 257-263.

Vermorken JB, Herbst RS, Leon X, et al. Overview of the

[22] efficacy of cetuximab in recurrent and/or metastatic squamous cell carcinoma of the head and neck in patients who previously failed platinum-based therapies [J]. Cancer, 2008, 112(12): 2710-2719.

[23 ] Martín-Martorell P, Roselló S, Rodríguez-Braun E, et al. Biweekly cetuximab and irinotecan in advanced colorectal cancer patients progressing after at least one previous line of chemotherapy: results of a phase II single institution trial [J]. Br J Cancer, 2008,99(3):455-458.

[24] Pfeiffer P, Nielsen D, Bjerregaard J, et al. Biweekly cetuximab and irinotecan as third-line therapy in patients with advanced colorectal cancer after failure to irinotecan, oxaliplatin and 5-fluorouracil [J]. Ann Oncol, 2008,19(6): $1141-1145$.

[25] Curran D, Giralt J, Harari PM, et al. Quality of life in head and neck cancer patients after treatment with high-dose radiotherapy alone or in combination with cetuximab $[\mathrm{J}]$. J Clin Oncol, 2007,25 (16):2191-2197. 